\title{
Braucht die Demokratie mehr städtische Autonomie?
}

\section{Zur politischen Philosophie der demokratischen Stadt}

\section{Political Philosophy and the City}

Narratives of urban democracy

\section{VERENA Frick, GöTtingen}

Zusammenfassung: Dieser Artikel nimmt die im Kontext einer vielfach diagnostizierten Renaissance der Stadt erhobene Forderung nach mehr städtischer Autonomie zum Ausgangspunkt, um aus demokratietheoretischer Perspektive der Frage nachzugehen, welche rechtfertigenden Gründe für eine größere städtische Autonomie angeführt werden können. Zu diesem Zweck rekonstruiert der Beitrag normative Leitbilder der demokratischen Stadt, die zugleich Lücken einer allein staatlich verstandenen Demokratiekonzeption verdeutlichen. Es handelt sich bei diesen Leitbildern um das Bild der Stadt als Schule der Demokratie, als urbane Kosmopolis sowie als urbane Allmende. Die untersuchungsleitende These lautet, dass die Stadt ein eigenständiger politischer Handlungsraum ist, an den spezifische demokratische Versprechen geknüpft sind und der spezifische Potenziale für die Verwirklichung einer demokratischen Praxis bietet. Das Erkenntnisinteresse ist zweigeteilt: Einerseits sollen in begrifflicher Perspektive die Leitbilder auf einen spezifisch urbanen Demokratiebegriff hin befragt werden und andererseits gilt es in systematischer Perspektive die zugrundeliegenden Vorstellungen von Gemeinschaft, Partizipation und Mitgliedschaft zu beleuchten und auf ihre demokratietheoretische Anschlussfähigkeit hin zu prüfen.

Schlagwörter: Stadt, Demokratie, Globalisierung, Mitgliedschaft, öffentlicher Raum

Abstract: The starting point of this article is the demand for more urban autonomy made in the context of a frequently diagnosed renaissance of the city. Adopting the perspective of democratic theory, it explores the question of which reasons may justify greater urban autonomy. To this end, the article reconstructs normative models 
of the democratic city and, at the same time, highlights gaps of a solely statist conception of democracy. In the following, three models are examined that assume the city as an independent political space. These are the model of the city as a school of democracy, as an urban cosmopolis, and as an urban commons. Discussing these models, the article argues that the city is a distinct space for political action, to which specific democratic promises are attached and which offers specific potentials for the realization of a democratic practice. The research interest is twofold: From a conceptual perspective, the article asks whether a specific urban concept of democracy exists, and from a systematic perspective, it disentangles the underlying notions of community, participation and democratic membership.

Keywords: city, democracy, globalization, citizenship, public space

\section{Einleitung: Die Renaissance der Städte als politische Akteure}

Stadtluft macht frei - dieses historische Emanzipationsversprechen der Stadt gewinnt heute neue Attraktivität. Das eingespielte Verhältnis von Stadt und Staat befindet sich im Wandel, Städte treten aus dem Schatten der sie umgebenden Nationalstaaten heraus und reklamieren politischen Gestaltungsanspruch. Besonders augenfällig ist das im Bereich der Klimapolitik, wo transnationale Städtenetzwerke wie die C40 Climate Cities Leadership Group voranschreiten und durch den Austausch von Informationen und Best-Practice-Lösungen sowie durch eigene Hilfsprogramme zur lokalen Realisierung der im Pariser Abkommen vereinbarten Klimaziele beitragen. Auch in anderen Bereichen sehen sich Staaten mit der politischen Aktivität der Städte konfrontiert. So haben Städte begonnen, sich als ,Menschenrechtsstadt' zu deklarieren, und sich auf teils höhere Menschenrechtsstandards, als sie auf nationaler Ebene gelten, verpflichtet (Oomen et al. 2016). Auch das in den USA in den 1980er Jahren entstandene sanctuary city movement findet inzwischen weltweit Nachahmung. Städte erklären sich zum Ort der Zuflucht für Geflüchtete und verweigern sich der umstandslosen Umsetzung nationaler Flüchtlingspolitik, indem sie etwa den Vollzug von Abschiebungen aussetzen oder Ausweisdokumente nicht kontrollieren (Boudou 2020).

Die Renaissance der Städte als politische Akteure hat in der öffentlichen Debatte eine regelrechte Euphorie ausgelöst. In den Feuilletons wird mit Blick auf die immer gelähmter wirkenden Nationalstaaten angesichts von Brexit, Handelskriegen, Klimawandel und Pandemien regelmäßig beschworen, dass die Probleme der Welt nun in den Städten gelöst werden müssen (Dettling 2019). Städte erscheinen dabei mal als Retter der UN-Ide- 
ale (Clüver Ashbrook und Haarhuis 2019), mal sollen sie den Weg aus der gegenwärtigen Krise der Demokratie weisen (von Borries 2019). Zu diesem neuen Glauben an die Veränderungskraft der Städte passen Selbstbilder prominenter Bürgermeisterinnen, die wie Anne Hidalgo einen neuen demokratischen Vertrag versprechen, in dem die Stadt zum „Ort des Möglichen“ wird (Hidalgo 2019, 37), oder die wie Rahm Emanuel die überlegene Innovationskraft der Städte und ihrer Bürgermeister gegenüber den nationalen Regierungen preisen (Emanuel 2020). Und ganz im Sinne einer engaged political philosophy hat auch Benjamin Barber (2013) ein leidenschaftliches Plädoyer dafür gehalten, warum Bürgermeisterinnen die Welt regieren sollten.

Die Stadt wird hier zu einem konkreten Ort der Hoffnung auf eine pragmatische und lösungsorientierte Politik, die an den kosmopolitischen Werten von Freiheit, Demokratie, Nachhaltigkeit, postnationaler Solidarität und offenen Grenzen orientiert ist. Einen beträchtlichen Teil ihrer neuen Attraktivität zieht die Stadt dabei aus dem in der Literatur häufig insinuierten Gegensatz zum Nationalstaat. Exemplarisch für die aktuelle Stadtdiskussion findet sich das bei Barber beschrieben (2013, 9ff.). Demnach orientiert sich staatliches Handeln vor allem am Paradigma der Souveränität, das heißt, Staaten zielen auf Unabhängigkeit und Autonomie, sie erweisen sich parteilich gegenüber der eigenen Nation und neigen zu Weltanschauungen und Ideologien. Ganz anders Städte: Aufgrund des lokalen, unmittelbaren Handlungsdrucks charakterisiert Barber städtisches Handeln als pragmatisch und problemlösend, unideologisch und innovativ. Oder wie es im Selbstbild vieler Städtenetzwerke heißt: states talk, cities act.

Städte sind umkämpfte Räume, in denen sich überörtliche Konflikte um soziale Ungleichheit, Migration oder Klimawandel, deren Lösung traditionell vor allem von Staaten erwartet wurde, sozialräumlich kristallisieren. Angesichts dessen wird die Diagnose einer gestiegenen politischen Bedeutung von Städten meist mit der Forderung nach größeren politischen Gestaltungsspielräumen für Städte verknüpft, um diese Konflikte lokal zu bearbeiten. Worin genau besteht dabei aber das demokratische Emanzipationsversprechen der Stadt im 21. Jahrhundert? Und woher beziehen Städte eigentlich das demokratische Mandat für den von ihnen reklamierten politischen Gestaltungsanspruch? Bei genauerem Hinsehen erinnern nämlich einige der oftmals als städtisch bezeichneten Attribute - Problemlösung, Sachorientierung, Pragmatismus - an den neoliberalen managerialism, der für globale Governance-Arrangements insgesamt charakteristisch ist und für seine Tendenz zur Entdemokratisierung weithin kritisiert wird (z. B. 
Koskenniemi 2007, 29). Leistet am Ende also eine gewisse Sehnsucht nach einfachem Entscheiden der großen Fragen unserer Gegenwart einer neuen Technokratie der Stadtverwaltungen Vorschub?

Diese Fragen zielen im Kern auf die Autorität und Legitimität städtischen Handelns im 21. Jahrhundert und verdeutlichen zugleich den Bedarf an einer politischen Philosophie der Stadt. Unstrittig scheint heute, dass eine politische Philosophie, die Städte lediglich als Teil des staatlichen Verwaltungsaufbaus begreift, der sich vorwiegend funktional durch eine effektive Erfüllung von Verwaltungsaufgaben legitimiert, wenig angemessen ist, um die politische Bedeutung städtischen Handelns zu rechtfertigen. Ein solches Verständnis wird jedoch in weiten Teilen der staatstheoretischen Literatur tradiert (kritisch: Wollmann 2002, 10) und liegt zumindest unausgesprochen auch den meisten Demokratietheorien zugrunde, die sich noch immer vor allem auf die staatliche Demokratie konzentrieren und über Städte kaum ein Wort verlieren. Damit fehlt es aber - zumindest in der deutschsprachigen politischen Philosophie - weitgehend an normativen Maßstäben einer demokratischen Stadt, die eine kritische Bewertung des städtischen Emanzipationsversprechens unter den Bedingungen des 21. Jahrhunderts ermöglichen würden. Zwar liegen inzwischen vielversprechende Einzelstudien zu stadtspezifischen Themen wie Gentrifizierung (Hofmann 2020; Huber und Wolkenstein 2018), Segregation (Sundstrom 2020; Bickford 2000) oder Städten als Orte des Widerstands (Sörensen 2020) vor, ein kohärentes Bild der demokratischen Stadt ergibt sich daraus indes noch nicht.

Vor diesem Hintergrund konzentriert sich der Beitrag auf die demokratietheoretischen Aspekte der Forderung nach mehr städtischer Autonomie gegenüber dem Staat. Im Mittelpunkt steht dabei die Frage, ob Städte mehr Gestaltungsspielräume erhalten sollten und welche demokratietheoretischen Gründe für die Legitimität städtischer Autonomie angeführt werden können. Zu dem Zweck werde ich den aktuellen Diskurs in der Stadtforschung auf die verstreuten Ansätze einer politischen Philosophie der Stadt durchmustern und in einem synthetisierenden Zugriff normative Leitbilder der demokratischen Stadt rekonstruieren. Die untersuchungsleitende These lautet, dass die Stadt ein eigenständiger politischer Handlungsraum ist, an den spezifische demokratische Versprechen geknüpft sind und der spezifische Potenziale für die Verwirklichung einer demokratischen Praxis bietet. Diese Versprechen, so möchte ich argumentieren, lassen sich zu drei in der politikphilosophischen Stadtdiskussion dominanten Leitbildern verdichten, die die Bedeutung des städtischen Raums für die Demokratie akzentuieren 
und zugleich auf Lücken einer allein staatlich verstandenen Demokratiekonzeption verweisen. Das Erkenntnisinteresse ist mit Blick auf die Leitbilder zweigeteilt: Einerseits sollen in begrifflicher Perspektive die Leitbilder auf einen spezifisch städtischen Demokratiebegriff hin befragt werden und andererseits gilt es in systematischer Perspektive die zugrundeliegenden Vorstellungen von Gemeinschaft, Partizipation und Mitgliedschaft zu beleuchten und auf ihre demokratietheoretische Anschlussfähigkeit hin zu prüfen. Damit versteht sich dieser Beitrag als Ansatz zu der noch ausstehenden Kartografie einer Demokratietheorie der Stadt, auf deren Basis die politische Philosophie erst Reformideen für eine Neubestimmung des Verhältnisses von Stadt und Staat formulieren und evaluieren kann.

Zwei Eingrenzungen sind dabei in definitorischer Hinsicht vorzunehmen: Aufbauend auf der klassischen Stadtdefinition der Chicago School entlang der drei Merkmale Größe, Dichte und soziale Heterogenität (Wirth 1938, 8) wird ,Stadt‘ im Folgenden als verdichteter sozialer Konfliktraum zwischen einander fremden Individuen verstanden (dazu auch Siebel 2018). Die damit implizierten Polarisierungen sind in der Regel in größeren Städten und Großstädten anzutreffen, sodass hier mit ,Stadt' vor allem Großstädte gemeint sind. Eine weitere Eingrenzung ist mit Blick auf die Reichweite und den Kontext der politischen Theorien vorzunehmen. Die folgenden demokratietheoretischen Überlegungen stehen im Kontext einer westlichen politischen Philosophie und der von ihr geprägten Begriffe. Deren transkulturelle Übertragbarkeit auf andere politische Kontexte kann nicht vorausgesetzt werden, sodass im weiteren Verlauf mit ,Stadt' nur Städte innerhalb liberal-westlicher Demokratien adressiert werden. Hier kann ferner vorausgesetzt werden, dass in der Regel durch Wahl legitimierte Selbstverwaltungsinstitutionen die Stadtpolitik bestimmen.

Im Folgenden werde ich in drei Schritten vorgehen: Zunächst verorte ich die Renaissance der Städte als politische Akteure im Kontext der Globalisierung und argumentiere, dass daraus eine neue Rechtfertigungsbedürftigkeit für städtisches Handeln resultiert (Abschnitt 2). Daran anschließend unterscheide ich drei normative Leitbilder der Stadt und befrage sie auf die zugrundeliegenden demokratischen Versprechen der Stadt und Vorstellungen politischer Mitgliedschaft (Abschnitt 3). Dazu zählt, erstens, die Vorstellung der Stadt als Schule der Demokratie, die der Entfaltung demokratischer Selbstwirksamkeit Raum gibt (3.1); zweitens, die Idee der urbanen Kosmopolis, die demokratische Mitgliedschaft auf Basis von Diversität und Differenz ermöglicht (3.2); und drittens, das Leitbild der urbanen Allmende, 
das den öffentlichen Raum der Stadt als Gemeingut und Basis eines ,Rechts auf Stadt' hervorhebt (3.3). Ein abschließender Vergleich der drei Leitbilder zielt auf die Beantwortung der Frage, inwiefern sie herangezogen werden können, um mehr städtische Autonomie zu legitimieren und welche Ordnungsvorstellungen des Verhältnisses von Stadt und Staat darin jeweils zum Ausdruck kommen. Zugleich sollen auf Basis des Vergleichs Desiderata einer Demokratietheorie der Stadt benannt werden (Abschnitt 4).

\section{Legitimationsprobleme globalen städtischen Handelns}

Die neue Rolle der Städte als politische Akteure steht in unmittelbarem $\mathrm{Zu}$ sammenhang mit der fortschreitenden Globalisierung und dem damit verbundenen Wandel von Staatlichkeit. Versteht man Globalisierung als weltweite Verdichtung und Entterritorialisierung sozialer, kultureller, politischer und ökonomischer Interaktionen, dann stechen die Öffnung des nationalstaatlichen ,Containers' und die Relativierung staatlicher Ordnungsmacht hervor. Der permanente Fluss von Waren, Ideen, Personen und Informationen macht nicht länger an nationalen Grenzen halt, sondern überwindet diese Grenzen sowohl materiell als auch virtuell durch digitale Kommunikationsmedien (Castells 2017). Während mit Blick auf diese Neuordnung der räumlichen Bezüge politischen und sozialen Handelns zunächst vor allem die transnationale bzw. internationale Ausdehnung betont wurde (Held 1997; Giddens 1990), zeigt sich inzwischen immer deutlicher das Bild einer parallel stattfindenden Lokalisierung globaler Entwicklungen. „Localities“ [...] serve nowadays as dumping grounds for problems generated globally not by their initiative and without their consultation“, diagnostizierte etwa Zygmunt Bauman (2013, 1f.) und prägte dafür den Begriff „glocalization“.

In Städten verdichten sich die globalen Herausforderungen der Gegenwart wie Klimawandel, soziale Ungleichheit, Migration und Pandemien, auf die damit auch eine lokale Antwort gefunden werden muss. Städte entwickeln sich auf diese Weise zu sogenannten „Bruchzonen der Globalisierung“, d.h. zu jenen Räumen, in denen sich die Neusegmentierung der Welt besonders deutlich zeigt und die Herstellung neuer Raumbezüge entsprechend umstritten ist (Middell 2009, 117). Besonders offensichtlich wird die steigende Relevanz der lokalen Ebene in den sogenannten global cities. Saskia Sassen (1991) hat als eine der Ersten auf das weltumspannende Netz von Metropolen wie Tokio, London, Frankfurt, New York, Singapur oder Johannesburg hingewiesen, in denen sich die weltweite Mobilität von Dienstleis- 
tungen, Kapital und Personen knotenpunktartig verdichtet. Als Standorte des internationalen Finanzkapitalismus entkoppeln sich die global cities, so ihre These, zunehmend vom Kontext der sie umgebenden Territorialstaaten und gleichen sich stattdessen untereinander immer weiter an.

Doch auch abseits der prägnanten global cities haben Städte weltweit begonnen, sich in transnationalen Netzwerken zu koordinieren. Besonders in den Bereichen Klima- und Umweltschutz, Kunst und Kultur, Sicherheit, Mobilität und nachhaltige Entwicklung ist inzwischen eine Vielzahl solch ,paradiplomatischer ${ }^{6}$ Zusammenschlüsse entstanden (Smith 2019). ${ }^{1}$ Und zahlreiche internationale Organisationen wie die Weltbank, die UN oder auch die Europäische Union haben ihrerseits die politische Bedeutung der Städte befördert. Immer häufiger suchen sie nach skalierten Lösungen, die gezielt unterschiedliche politische Handlungsebenen ansprechen sollen, und überspringen dabei die staatliche Ebene, um direkt die Städte zu adressieren. Angesichts dessen ist bereits davon die Rede, dass eine „novel trinity: World - State - Locality“ (Blank 2006, 889) die Staatendominanz der internationalen Ordnung relativiert.

Der Aufstieg der Städte ist Teil der umfassenderen Rekonfiguration staatlicher Herrschaft, die die Globalisierung eingeleitet hat (Frick 2020, 69). Städte partizipieren dabei an einem Prozess, der als Auffächerung oder Disaggregation staatlicher Souveränität beschrieben wird (Volk 2019; Cohen 2014; Brown 2010; Grimm 2009; Slaughter 2004). Gemeint ist damit, dass die Globalisierung die strikten Grenzziehungen zwischen innen und außen, staatlich und international, auf denen das westfälische System der Staatenordnung aufgebaut war, unterläuft und bisweilen sogar auflöst. An die Stelle einer hierarchischen, auf dem Prinzip der Staatensouveränität aufgebauten internationalen Ordnung ist ein heterarchisches Regieren in internationalen Institutionen und transnationalen Netzwerken getreten. Ein Effekt der Netzwerk-Governance ist, dass sie neue Handlungsräume für politische Akteure jenseits des Staates eröffnet (Volk 2019; Aust 2017; Nijman 2016; Acuto 2013). Innerhalb der Netzwerke macht eine Vielzahl öffentlicher und privater Akteure den Staaten ihr Monopol auf politische Gestaltung streitig. Zwar werden in diesen Netzwerken - das zeigt auch die Praxis der transnationalen Städtenetzwerke - nicht immer rechtsverbindliche Entscheidungen getroffen. Doch auch der indirekte Zwang, den Best-Practice-Beispiele, Zielver-

1 Eine Aufstellung der zahlreichen Netzwerke findet sich bei Barber (2013, $118 \mathrm{ff}$.). 
einbarungen, Compliance-Zusagen, Fördergelder und Monitoring-Verfahren auf die beteiligten Akteure ausüben, entfaltet eine handlungssteuernde Wirkung, da er die politischen Kosten für abweichendes Verhalten erhöht.

Mit Blick auf die demokratierelevanten Konsequenzen und besonders die Frage nach der normativen Anerkennungswürdigkeit der entstehenden globalen Governance-Ordnung fällt das Urteil ambivalent aus: Einerseits steckt in den Netzwerken demokratisches Emanzipationspotential, man denke hier nur an transnationale Protestbewegungen und NGOs, die sich globalen Problemen verschrieben haben und über die Grenzen des Staates hinweg Unterstützerinnen mobilisieren können. Auf diese Weise entsteht die Möglichkeit, Themen wie Klimagerechtigkeit, Artensterben, Welthandel oder Armut zu politisieren, die die Menschheit als Ganzes betreffen und innerhalb eines einzelnen Nationalstaates kaum adäquat adressiert werden könnten. Die Vorstellung einer globalen res publica kann dadurch überhaupt erst hervorgebracht werden (Volk 2019, 21).

Andererseits ist die demokratische Legitimation der neuen Governance-Strukturen in vielen Fällen prekär (Zürn 2011, 67; Nullmeier und Nonhoff 2010, 16). Zugespitzt zeigen das private Akteure des Finanzkapitalismus wie global agierende Unternehmen, Anwaltskanzleien oder Rating-Agenturen, deren Einfluss auf die Politikgestaltung in den Governance-Netzwerken beträchtlich ist, ohne dass sie hierfür demokratisch autorisiert wären. Doch auch innerhalb der demokratischen Staaten drohen supranational getroffene Vereinbarungen etwa auf der Ebene der Europäischen Union die demokratischen Verfahren auszuhöhlen, da diese Entscheidungen dem demokratischen Willensbildungsprozess weitgehend entzogen sind und von den nationalen Parlamenten nur noch im Ganzen ratifiziert werden können (Habermas 2011, 51). Zwar wurde versucht, auf die Legitimationsprobleme politischer Herrschaft jenseits des Staates mit einer Neufassung des Legitimitätsbegriffs entlang liberaler Output-Kriterien wie Problemlösung, Expertise oder Schutz von Individualrechten zu reagieren (Nullmeier und Nonhoff 2010, 28). Dieser Wandel des Legitimitätsdenkens vermag indes nicht zu überdecken, dass die Output-Orientierung nur um den Preis des republikanischen Versprechens auf gleiche und effektive Teilhabe an der demokratischen Selbstregierungspraxis zu haben ist (Jörke 2019, 29).

Städte sind von dieser grundsätzlichen demokratietheoretischen Kritik an den neuen Akteuren und Netzwerken der Global Governance nicht ausgenommen. Ganz im Gegenteil, folgt man der klassischen Vorstellung demokratischer Legitimität, in deren Mittelpunkt die Volkssouveränität steht, 
dann trägt das grenzüberschreitende politische Handeln von Städten nur dazu bei, demokratische Souveränität weiter zu unterminieren. Denn aus dieser Perspektive besteht ein enger begrifflicher und systematischer $\mathrm{Zu}$ sammenhang zwischen Volks- und Staatensouveränität. Die demokratischen Verfahren und Meinungsbildungsprozesse innerhalb des demokratischen Staates geben der „Selbsteinwirkung einer politisch organisierten Bürgergesellschaft“ Raum und gewährleisten, dass sich die Bürgerinnen zugleich als Adressaten und Autoren bindender Gesetze verstehen dürfen (Habermas 2011, 50). Das Volk der Staatsbürgerinnen konstituiert das einheitliche Legitimationssubjekt für die Ausübung staatlicher Souveränität, die gewissermaßen nur die nach außen gewendete Seite demokratischer Selbstbestimmung ist und den politischen Handlungsspielraum reflektiert, dessen die effektive Realisierung kollektiver Handlungsmacht bedarf (Böckenförde 1991).

Die darin zum Ausdruck kommende Container-Vorstellung des politischen Raums im Nationalstaat basiert auf einer binären Trennung zwischen innen und außen: Während sich im Inneren des Staates der Wille des Volkes in einem diskursiven Verfahren erst konstituiert, vertritt der Staat nach außen als Einheit die Ergebnisse der demokratischen Selbstbestimmungspraxis. Beginnen nun substaatliche Einheiten wie Städte durch eigenes grenzüberschreitendes Handeln diese Differenz zu unterlaufen, kann das unter Zugrundelegung des Souveränitäts-Paradigmas in zweierlei Hinsicht bewertet werden: Entweder als potentieller Widerspruch zu den demokratischen Entscheidungen des einheitlichen demos oder als Praxis einer internationalen Behördenkooperation, innerhalb derer die Stadt in eher technokratisch-funktionaler Perspektive als behördliches Abbild des Gesamtstaats en miniature agiert (Aust 2017, 182). Im Ergebnis lassen jedoch beide Varianten nur eine Schlussfolgerung zu, nämlich die Zurückweisung eines eigenen demokratischen Mandats von Städten als illegitime Selbstermächtigung.

Dieses Denken von der Souveränität her liegt implizit oder explizit dem Mainstream der politischen Philosophie und Demokratietheorie zugrunde und behindert die Wahrnehmung von Städten als eigenständige demokratische Räume. Bei genauerem Hinsehen zeigt sich jedoch, dass die Zentralität des Souveränitätsparadigmas immer schlechter plausibilisiert werden kann (Rosanvallon 2010). Die ihm zugrunde liegende Einheitsfiktion wird in ihrer zentralen Dimension der Einheit von Volk, Staatsgewalt, Territorium und Recht von einer anschwellenden Forschungsliteratur zu postnationalen Vorstellungen des demos (Patberg 2018), disaggregierter Staatsgewalt (Slaughter 2004) und globalem Rechtspluralismus (Krisch 2012) in Frage 
gestellt. Kleinster gemeinsamer Nenner dieser Ansätze ist die Überzeugung, dass sich das Souveränitätsparadigma sowohl normativ als auch empirisch in zunehmendem Maße als inadäquat erweist, die politische Realität in der ,postnationalen Konstellation' (Habermas) zu erfassen.

Der Fokus ist jedoch überwiegend auf inter- und transnationale Prozesse gerichtet (Fraser 2010). Was dabei übersehen wird, ist die gleichzeitige Rekonfiguration innerstaatlicher politischer Ordnungsbildung, die ebenfalls neue Legitimationsfragen stellt. In der stark unitaristisch ausgerichteten Demokratietheorie setzt sich erst allmählich, insbesondere unter dem Eindruck der Globalisierung, ein Bewusstsein für unterschiedliche scales der Demokratie durch (Brenner 2019; Fraser 2010; Sassen 2007). Gemeint ist damit nicht eine hierarchische Mehrebenenarchitektur, sondern ein Denken in sich teils überlappenden, teils distinkten sozial konstituierten Handlungsräumen, zwischen denen durch die Öffnung des nationalstaatlichen,Containers ${ }^{6}$ neue Bezüge möglich werden. Städte nehmen an dieser Rekonfiguration politischer Herrschaft teil. Insbesondere für die Beziehung zwischen Stadt und Staat ist diese Entwicklung folgenreich: Sie versetzt Städte in die Lage, die sich eröffnenden politischen Gestaltungsspielräume nicht mehr nur innerhalb der ihnen staatlich zugewiesenen Aufgaben, sondern zumindest potentiell in Konkurrenz bzw. Konflikt mit dem Staat wahrzunehmen. Dies zeigen etwa die Beispiele der selbsterklärten sanctuary cities, human rights cities oder der Null-Emissions-Städte, die über staatliche Verpflichtungen in den Bereichen Migration, Menschenrechts- und Klimaschutz hinausgehen und damit den Staat in diesen Politikfeldern unter Handlungsdruck setzen. Städte sind damit nicht länger Ebenen „ausgegliederter Konfliktabsorption“, die im stabilitätspolitischen Eigeninteresse den Zentralstaat gegenüber lokalen politischen Forderungen immunisieren, wie Claus Offe $(1975,308)$ einmal argumentierte. Die politische Bedeutung der Stadt kann heute nicht mehr ausschließlich vom Staat her erfasst werden.

\section{Städte als demokratische Räume}

In wessen Namen aber handeln Städte, gerade wenn sie sich in Opposition zum Staat begeben? Dies ist die Frage nach einem genuin städtischen demos und damit nach einem distinkten städtischen Raum politischer Mitgliedschaft jenseits des Staates. Zugleich verdeutlicht diese Frage die politikphilosophische Notwendigkeit, über die demokratische Legitimität städtischen Handelns und die Verortung von Städten innerhalb der staatlichen 
Legitimationsarchitektur der liberalen Demokratie neu nachzudenken. Dazu muss die politische Philosophie die black box der Stadt öffnen und nach den rechtfertigenden Gründen für die normative Anerkennungswürdigkeit städtischer Autonomie fragen. Wenn ich im Folgenden argumentiere, dass Städte eigene demokratische Handlungsräume konstituieren, an die spezifische demokratische Versprechen geknüpft sind, dann soll damit nicht geleugnet werden, dass Städte immer auch Teil des staatlichen Verwaltungsapparates sind und sich funktional im Sinne einer effektiven Erfüllung von Staatsaufgaben rechtfertigen müssen. Doch die politische Bedeutung von Städten erschöpft sich nicht in Problemlösungskompetenz und Verwaltungsexpertise. Vielmehr eröffnen Städte einen Raum politischer Gemeinschaftsbildung, sie sind in der Lage, eine politische Gemeinschaft zu konstituieren, deren Mandat sie als politische Akteure wahrnehmen. Was macht diesen Raum aber zu einem demokratischen Raum? Und worin besteht das demokratische Potenzial bzw. das Emanzipationsversprechen der Stadt im 21. Jahrhundert? Nach der Analyse der gegenwärtigen politikphilosophischen und interdisziplinären Stadtdiskussion schlage ich drei normative Leitbilder der demokratischen Stadt vor, die jeweils unterschiedliche Vorstellungen von politischer Mitgliedschaft in der städtischen Demokratie akzentuieren und entsprechend unterschiedliche Schlussfolgerungen für das Verhältnis von Stadt und Staat zulassen. Im Mittelpunkt steht dabei die Frage, welche Vorstellungen der städtischen Demokratie in den Leitbildern zum Ausdruck kommen und inwiefern sich auf Basis dieser Vorstellungen die Renaissance der Städte als politische Akteure demokratietheoretisch rechtfertigen lässt.

In erkenntnistheoretischer Hinsicht erfüllen die Leitbilder eine heuristische Funktion und sind als ein Angebot zu verstehen, die verstreute Stadtdiskussion zu ordnen und zu systematisieren. Dabei erheben die Leitbilder nicht den Anspruch, einen jeweils kohärenten Ansatz einer politischen Philosophie der Stadt abzubilden - ein solcher existiert ohnehin, soweit ersichtlich, derzeit noch nicht. In ihnen verdichten sich vielmehr mitunter heterogene Ansätze, die aber zentrale Prinzipien und Ziele gemeinsam haben und sich darin von anderen Ansätzen unterscheiden. Damit ist nicht gesagt, dass sich die Leitbilder wechselseitig ausschließen, sie lassen sich mit Blick auf ihre Prämissen und normativen Zielvorstellungen indes analytisch unterscheiden: Während das Leitbild der Stadt als Schule der Demokratie auf eine spezifisch städtische Funktion abhebt, beschreibt das Leitbild der urbanen Kosmopolis eine moralische Praxis der Stadtbürgerinnen. Das Leitbild der Stadt als Allmende nimmt wiederum Bezug auf Eigentumsrechte und 
Praktiken des commoning. Die Leitbilder zeichnen sich durch ihre narrative Dimension aus, sie entwerfen ausgehend von einer Krisendiagnose eine alternative Erzählung davon, was ,Stadt' ausmacht, verbinden insofern empirische Diagnosen mit normativen Ansprüchen und legitimieren Forderungen nach einer veränderten Stadtpraxis. Vor diesem Hintergrund dienen die hier vorgestellten Leitbilder als doppelter Ausgangspunkt, einerseits, um eine politische Philosophie der Stadt zu entwickeln, und andererseits, um stadtspezifische Fragen im Kontext der vorwiegend auf den Staat bezogenen politischen Philosophie und Demokratietheorie zu diskutieren und auf diese Weise die blinden Flecke der gegenwärtigen politischen Philosophie mit Blick auf die Stadt zu erhellen.

\subsection{Die Stadt als Schule der Demokratie}

Von Tocqueville über Mill hat die Vorstellung von der Stadt als Schule der Demokratie eine lange Tradition in der politischen Ideengeschichte der Moderne. In jüngerer Zeit findet sich der Gedanke einer demokratiestärkenden Dialektik aus lokaler politischer Aktivität und politischer Gemeinschaftsbildung vor allem bei Autoren, die dem Republikanismus bzw. dem Kommunitarismus zugerechnet werden. Dezentralisierung, Subsidiarität und Föderalismus als politische Ordnungsprinzipien werden hier an die Erwartung politischer Aktivierung und bürgerschaftlichen Engagements gekoppelt und gegen die Entfremdung der Individuen voneinander und von ihrem Gemeinwesen in der liberalen, kapitalistischen Demokratie exponiert. Die städtische Ebene soll „den geeigneten Rahmen einer geteilten sozialen Praxis stiften“ (Haus 2002, 91) und damit jene gemeinsame Lebenswelt aufrechterhalten, die die Basis ist für die doppelte Transformation atomisierter Individuen $\mathrm{zu}$ verantwortlichen Staatsbürgerinnen und privater Interessen zu öffentlichen Angelegenheiten, wie sie im Zentrum republikanisch-partizipatorischer Demokratietheorien steht (Barber 2004 [1984], 119). Diese anspruchsvolle Demokratietheorie baut auf ein starkes Gemeinschaftsgefühl zwischen den Bürgern auf, das wiederum selbst erst durch Praxis und Erfahrung hergestellt werden muss. Und genau hier zehrt die Demokratie von den Möglichkeiten städtischer Gemeinschaftsbildung.

Dieser Zusammenhang lässt sich exemplarisch anhand von Benjamin Barbers „starker Demokratie“ (2004 [1984]) und Hartmut Rosas Überlegungen zum Verhältnis von Resonanz und Demokratie (Rosa 2019) verdeutlichen. Beide vereint ein Denken von der menschlichen Erfahrung her, das die Trennung zwischen dem Menschen als Sozialwesen und als po- 
litischem Wesen aufhebt und einen entsprechend weiten Politikbegriff voraussetzt, der das Politische im Zusammenleben der Menschen selbst statt in formalisierten Entscheidungsverfahren verortet. Barber geht von der Prämisse der grundsätzlichen Konflikthaftigkeit menschlichen Zusammenlebens aus, die aber anders als in der liberalen politischen Philosophie John Rawls' nicht durch die Anerkennung einer politischen Gerechtigkeitskonzeption beigelegt werden kann, sondern durch die Ausbildung politischer Urteilskraft durch citizenship und Partizipation (Barber 2004 [1984], 157f.). Gemeinschaftsbildung wird hier als Antwort auf das Problem politischer Ordnung formuliert. Barber hat dabei eine Vorstellung von Bürgersolidarität vor Augen, die sich am Ideal demokratischer Nachbarschaft orientiert: „Citizens are neighbors bound together neither by blood nor by contract but by their common concerns and common participation“ (ebd., 219). Lokale Institutionen wie Bürgerversammlungen bezeichnet er entsprechend als „training ground for democracy“ (ebd., 235). Ein ähnlicher Gedanke findet sich in Hartmut Rosas resonanztheoretischer Bestimmung der Demokratie (Rosa 2019). Obwohl in Rosas Überlegungen keine explizite Theorie der Stadt enthalten ist, lässt sich ausgehend von seinem republikanischen Demokratieverständnis ein Argument für die demokratische Bedeutung der lokalen Ebene entwickeln. In Anlehnung an Hannah Arendt sieht Rosa das Grundversprechen der Demokratie darin, mittels gemeinsamer politischer Gestaltung die soziale Welt und den öffentlichen Raum als Resonanzsphäre zu erleben. Die Bürgerinnen müssen sich darin mit ihren Wertvorstellungen wiederfinden, um die soziopolitische Ordnung als ihre eigene zu erkennen (ebd., 168). Prägend sei dabei die Erfahrung demokratischer Selbstwirksamkeit, d.h. durch Handeln individuell oder kollektiv etwas bewirken und andere damit erreichen zu können. Ein solches interpersonales Verständnis demokratischer Selbstwirksamkeit ist auf physisch-bürgerschaftliche Interaktions- und Begegnungsräume als „Resonanzsphäre“ angewiesen, in denen „sich die unterschiedlichen Stimmen artikulieren, sich wechselseitig hören und antworten und dadurch transformieren können“ (ebd., 185, 187). Die Betonung von Präsenz, Erfahrung und Erleben verweist auf die besondere Rolle des Lokalen für ein resonantes Weltverhältnis. Rosa zufolge ist nicht die Reichweite unserer Weltbeziehung entscheidend, sondern deren Qualität (Rosa 2020, 725).

Auffallend ist, dass sowohl Rosa als auch Barber den Aspekt der Gemeinschaftsbildung und der horizontalen Interaktion zwischen Bürgerinnen betonen. Mit Michael Haus kann man sagen, dass sie der lokalen Politik die 
Funktion einer „Politikermöglichungs-Politik“ (Haus 2002, 95) zuweisen: Nicht kollektive Entscheidungsfindung oder die Selbstregierung im engeren Sinne stehen hier im Vordergrund, sondern die gesellschaftstheoretischen Voraussetzungen und das soziale Kapital für die Verwirklichung und Stabilisierung einer demokratischen Ordnung. Das politische Engagement auf lokaler und nachbarschaftlicher Ebene dient mithin der Befähigung der Bürger und der Ausbildung demokratischer Urteilskraft und nicht der Machtausübung; so heißt es etwa bei Barber: „The objective is not yet to exercise power or make policy: it is to create the conditions for the exercise of power - to instill civic competence." (Barber 2004 [1984], 268) Als Schule der Demokratie ist die Stadt gewissermaßen eine lokale Antwort auf die Krise der liberalen Demokratie, wie sie sich im globalen Erstarken des Populismus offenbart.

Barber vollzieht später einen Kurswechsel: Noch in seiner ,starken Demokratie 'lehnt er die Eigenständigkeit lokaler Institutionen als potenziell demokratiezersetzenden Parochialismus ab (Barber 2004 [1984], 235). Rund 30 Jahre später bezeichnet er dagegen Städte als Bausteine einer „global democratic architecture“ (Barber 2013, 42) und engagiert sich auch politisch für ein Weltparlament der Bürgermeister. Barbers Plädoyer für eine aktive Rolle der Städte bei der Gestaltung einer demokratischen Global Governance hat viel mit einer spürbaren Ernüchterung über die Problemlösungsfähigkeit und Progressivität des Nationalstaates zu tun. ${ }^{2}$ In seinen demokratietheoretischen Überlegungen bleibt Barber indes, wie auch Rosa, stets auf die staatliche politie gerichtet. Beide denken politische Gemeinschaft in erster Linie im staatlichen Rahmen. Und beide entwickeln keinen städtischen Demokratiebegriff, sondern beschreiben vielmehr eine lokale Praxis, die nicht

2 An dieser Stelle kann nicht ausführlich auf theorieimmanente Kontinuitäten und Brüche eingegangen werden. Angesichts der globalisierungsbedingten Öffnung des staatlichen ,Containers' sieht Barber in zuvor ungekannter Weise die Chance, städtische Gemeinschaftsbildung mit der Lösung globaler Probleme zu verknüpfen (Barber 2013, 5). Zu diesem Bild der Stadt als Problemlöserin passt, dass Barber mit Effizienz und Pragmatismus vor allem Rechtfertigungsnarrative bemüht, die nicht genuin demokratische Qualität haben. Das verdeutlichen auch die in If mayors rule the world enthaltenen Porträts prominenter Bürgermeisterinnen, wobei Barber selten eine besonders inklusive und responsive demokratische Praxis der Stadtspitze beleuchtet, sondern in personalisierter Weise effizientes Entscheiden und Leadership-Qualitäten hervorhebt. 
exklusiv an den städtischen Raum gebunden ist. ${ }^{3}$ Ihr Akzent auf zwischenmenschlicher, nachbarschaftlicher Verbundenheit lässt vor allem an kleine Kommunen denken und passt nur bedingt zu der Anonymität, Blasiertheit und Oberflächlichkeit, die seit Georg Simmels (1995 [1903]) klassischer Beschreibung mit „urbanism as a way of life“ (Wirth 1938) verbunden werden. Die Bedeutung der lokalen Politik ist vielmehr auf einem Kontinuum hin zur gesamtstaatlichen Ebene angesiedelt, deren demokratische Qualität gleichwohl von der Unterstützungsleistung lokaler Praxis zehrt.

\subsection{Die urbane Kosmopolis}

Während in der republikanischen Vorstellung der Stadt als Schule der Demokratie die horizontale Vergemeinschaftung der Bürgerinnen im Vordergrund steht, mit anderen Worten aus Fremden Nachbarn werden sollen, rückt das Leitbild der urbanen Kosmopolis das städtische Versprechen auf Fremdsein und Fremdbleiben in den Fokus. Gerade aufgrund der oben genannten Anonymität, Blasiertheit und Oberflächlichkeit großstädtischen Lebens erscheinen Städte in dieser Perspektive als soziale Räume, innerhalb derer „unassimilated otherness“ (Young 1986, 22) überhaupt erst möglich werden kann. Diese Eigenschaften bilden im Leitbild der Stadt als Kosmopolis die gesellschaftstheoretische Basis für die normative Anerkennung von Differenz sowie eine politische Praxis der Differenz. Auf diese Weise wird die Stadt zum Ermöglichungsraum eines neuen Narrativs demokratischer Mitgliedschaft, das als postnationales „urban citizenship“ bezeichnet wird (Bauböck 2020; 2003). Dahinter steht die Erwartung, dass sich in der Stadt eine kulturelle, politische und rechtliche Praxis der Mitgliedschaft auf Basis von Heterogenität und Diversität realisieren kann. Wo Nationalstaaten eher zögerlich auf eine immer diversere Bürgerschaft reagieren, sollen Städte Vorreiter einer „cosmopolitan transformation of national conceptions of membership from below and from within“ (Bauböck 2003, 156) werden und auf die globale Herausforderung durch Flucht und Migration eine lokale Antwort geben.

Der städtische Raum scheint dabei besser geeignet, eine egalitäre Praxis auf der Basis von Heterogenität und sozialen Differenzen zu eröffnen als die staatliche politische Gemeinschaft, die sich noch immer schwertut,

3 Das verdeutlichen auch die von Rosa als Beispiel für die Wiedererlangung von Selbstwirksamkeit angeführten Praktiken wie die Gründung lokaler Energiegenossenschaften, die sich nicht ausschließlich in Städten finden. 
politische Mitgliedschaft jenseits nationaler Identität zu denken. Die Soziologie zählt Fremdsein traditionell zu den Grunderfahrungen der städtischen Lebensweise. Auch wenn heute eine zunehmende Angleichung der Lebensstile zwischen Stadt und Land konstatiert wird, bleibt Fremdsein in den von Migration und Diversität ungleich stärker geprägten Städten eine primär städtische Erfahrung. Stadtsoziologen wie Walter Siebel haben daher Fremdheit zum definierenden Merkmal der zeitgenössischen Stadt erhoben. Stadt ist demnach „ein Ort, an dem Fremde leben“ (Siebel 2018, 286). Diese Diagnose nehmen Autorinnen wie Iris Marion Young, Rainer Bauböck oder Ash Amin, deren Ansätze hier unter dem Leitbild der urbanen Kosmopolis versammelt werden, als Ausgangspunkt, um ein normatives Ideal der „unoppressive city“ (Young 1986, 20) zu entwickeln. Fremdsein erscheint darin nicht als zu überwindender Zustand auf dem Weg politischer Gemeinschaftsbildung, sondern als Voraussetzung einer „social differentiation without exclusion“, in deren Rahmen der normative Eigenwert von Differenz betont wird (ebd. 1990, 238). Insinuiert wird, dass in der Stadt - anders als auf nationalstaatlicher Ebene, wo Integration meist auf den Abbau von Differenz zielt - eine gesellschaftspolitische Inklusion möglich ist, die Differenz unangetastet lässt (Amin 2002, 972).

Dass dies in der Stadt erreichbar sein soll, hat vor allem mit der räumlichen Nähe der Bewohnerinnen zu tun. Die physische Nähe lässt einen geteilten Problem- und Erfahrungshorizont der Stadtbürger entstehen und konstituiert gemeinsame Betroffenheit. Die räumliche Nähe der Stadtbewohnerinnen überlagert gewissermaßen ihre soziale Distanz und bildet Young zufolge das moralische Fundament jener "differentiated solidarity“, die eine die wechselseitige Differenz affirmierende und respektierende Praxis ermöglichen soll (Young 2000, 221). Mit dem Konzept der „differentiated solidarity" beschreibt sie ein Ideal sozialer und politischer Inklusion, das sich, aufbauend auf gegenseitigem Respekt und wechselseitiger Verpflichtung zur Achtung der Differenz, gegen Exklusion und Segregation wendet und dabei individuelle und gruppenspezifische Partikularität zulässt (ebd.).

Ein solches Stadtleben als „the ,being-together" of strangers“ (Young 1986, 21) ist normativ ebenso anspruchsvoll wie voraussetzungsreich. Es setzt vor allem die Inklusivität des städtischen Raums als eines gemeinsamen Erfahrungs- und Erlebnisraums voraus. Intendiert ist damit nicht allein ein moralisches Argument für öffentliche Räume wie Plätze, Parks oder Bibliotheken, sondern eine Achtsamkeit gegenüber der räumlichen Manifestation von Differenz. Als moralisch legitim anerkannt wird in dieser Perspektive ein 
gruppenspezifisches Bedürfnis nach „social group distinction“, das aber nicht $\mathrm{zu}$ einer potenziell unüberwindbaren räumlichen Segregation auf Basis sozialer oder ethnischer Merkmale führen darf (Young 2000, 224; Sundstrom 2020, 224; Bauböck 2003, 145). Der aktive Abbau sozialer Schranken - etwa in Bezug auf die Anbindung an den öffentlichen Nahverkehr, den Zuschnitt von Schulbezirken oder den Zugang zu kulturellen Einrichtungen - ist dann nur die andere Seite eines legitimen Rechts auf freiwillige Selbst-Separierung.

Auch die normativen Erwartungen an die Stadtbürgerinnen der urbanen Kosmopolis sind hoch. Das vermeintliche Ideal von gemeinsamen Werten und Gemeinschaftsbildung ist aus der Perspektive dieses Leitbildes nicht nur unerwünscht, sondern wird als strukturell diskriminierend und unterdrückend abgelehnt (Young 1986, 12). Stattdessen bedarf es der konstanten „negotiation of difference“ (Amin 2002, 959) im alltäglichen, konflikthaften Zusammenleben der Individuen und Gruppen. Geteilte Werte entstehen hier allenfalls als temporäre, eher zufällige „accidents of engagement“ (ebd., 972). Sowohl in Amins agonistischer Perspektive auf Stadtpolitik als auch in Youngs Ideal der „unoppressive city“ müssen die einzelnen Stadtbürger die Zumutungen des Fremdseins nicht nur aushalten, sondern Differenz willkommen heißen und die Bereitschaft mitbringen, sich am tagtäglichen Prozess des oftmals konfliktreichen Aushandelns von Differenz zu beteiligen (Young 1986, 22; Amin 2002, 973).

Um den Zugang zu diesem Prozess möglichst offen zu gestalten und damit das spezifisch städtische Potential gesellschaftlicher Konfliktbearbeitung auch politisch zu institutionalisieren, hat Bauböck die Anerkennung eines eigenen ius domicilii als formelles Recht auf Stadtbürgerschaft vorgeschlagen (Bauböck 2020; 2003). Kern dieses urban citizenship ist ein mit dem Wohnsitz verknüpftes Recht auf städtische Mitgliedschaft, das unabhängig von der Staatsbürgerschaft automatisch durch Anmeldung eines festen Wohnsitzes erworben werden soll und damit auch Migrantinnen offensteht. An Migranten wie autochthone Bevölkerung würde das ius domicilii, so Bauböck (2003, 150), ein symbolisches Zeichen der Zugehörigkeit senden und die Erwartung verdeutlichen, die damit verbundenen Partizipationsrechte auch zu nutzen; zugleich würde die Stadtbürgerschaft die Stadt formell als eigenständiges politisches Gemeinwesen konstituieren, das sich auf einen städtischen demos stützt.

Dahinter scheint wiederum die Erwartung auf, dass Städte, Großstädte zumal, die besten Voraussetzungen dafür bieten, Differenz nicht nur auszuhalten, sondern als produktiv und bereichernd zu erleben. In der Stadt, 
so die Hoffnung, kann sich auf diese Weise das Ideal der kosmopolitischen Weltbürgerin räumlich manifestieren und zu einer postnationalen „democratic integration within and beyond nation-states“ (Bauböck 2020) beitragen. Städte als „homebase for cosmopolitan democracy“ (Bauböck 2003, 157) erscheinen hier als Vorreiterinnen einer Transformation des überkommenen, die nationale politische Gemeinschaft voraussetzenden Demokratieverständnisses hin zu einer postnationalen Demokratie, die das Recht von Individuen und Gruppen auf Differenz verwirklicht.

\subsection{Die urbane Allmende}

Im Leitbild der urbanen Kosmopolis erscheint die Stadt als Ermöglichungsraum eines individuellen und gruppenspezifischen Rechts auf Differenz. Auch das Leitbild der urbanen Allmende bekräftigt bestimmte Rechtspositionen, die an den städtischen Raum gebunden sind. Anders aber als die kosmopolitische Vision einer diskriminierungsfreien Stadt, die stärker in der liberalen Tradition eines negativen und individualistischen Freiheitsdenkens steht, konzentriert sich das Leitbild der urbanen Allmende auf die sozioökonomische Gleichheitsdimension der Demokratie und die räumliche Manifestation sozialer Ungleichheit im neoliberalen Kapitalismus. Ausgangspunkt ist dabei die Beobachtung, dass die durch den globalen Kapitalismus verursachte ungleiche Verteilung von Lebenschancen durch Privatisierung, Gentrifizierung und damit verbundene lebensweltliche Fragmentierung gleichsam in den städtischen Raum eingeschrieben ist und das Recht auf gleiche Teilhabe und Chancengleichheit zu untergraben droht. Anknüpfend an Henri Lefebvres vielzitiertes „Recht auf Stadt“ (2019 [1968]) haben Autorinnen wie Margaret Kohn sowie Vertreterinnen der critical urban studies die Vorstellung der Stadt als Allmende entwickelt und damit weitreichende Dekommodifizierungen und Mitspracherechte an der Stadtgestaltung begründet. Im Kampf um „local justice“ wird die Stadt hier als Gemeingut verstanden, auf das alle Stadtbewohner ein Anrecht haben (Kohn 2016, 8; Davies et al. 2010). Der Begriff der Allmende bezeichnet gemeinhin vor allem natürliche Ressourcen wie Boden, saubere Luft, Jagd- und Fischbestände oder Wälder, die allen Menschen gleichermaßen zugutekommen und die nur schwer kommodifizierbar sind (Ostrom 1990). Ihrem Charakter als Gemeineigentum entsprechen gleiche Nutzungs- und Zugangsrechte aller Menschen bzw. aller Mitglieder einer politischen Gemeinschaft (Siehr 2016, 667). Diesen Gedanken hat Lefebvre auf die Stadt übertragen und das Urbane als „das Werk der Städter“ (Lefebvre 2019 [1968], 105) bezeichnet. Die Stadtbewoh- 
nerinnen erschaffen sich, so Lefebvre, aufgrund der Unmittelbarkeit des Gelebten in der Stadt eine eigene Welt, eine „einzigartige Totalität“, die auf Begegnung, Gleichzeitigkeit, Zusammenwirken und Differenz beruht (ebd., 104; 45). Das Urbane wird hier zu einer zwischenmenschlichen Qualität, die sich im städtischen Raum entfaltet und ein „Recht auf städtisches Leben“ begründet (ebd., 166).

Doch ebenso wie die natürlichen Allmende-Güter bleibt auch die Stadt nicht von der sogenannten „tragedy of the commons“ - d.h. der Ausbeutung der Gemeingüter durch Maximierung des Eigeninteresses (Hardin 1968) - verschont. Die in der Tradition des Marxismus stehenden critical urban studies machen dafür vor allem die politische Ökonomie des Kapitalismus verantwortlich (Harvey 2016). Lefebvre betrachtete Industrialisierung und Fordismus als ursächlich für die Zerstörung des Urbanen, heute werden die Immobilienwirtschaft und die Privatisierung des öffentlichen Raums, eine zunehmend unternehmerisch denkende Stadtverwaltung sowie die an einer monetären Verwertungslogik orientierte Ausrichtung des Stadtmarketings auf creative city, Festivalisierung und Musealisierung als Bedrohung der urbanen Allmende kritisiert (Häußermann et al. 2018, 268; Harvey 2016; Holm 2011).

Gegen die kapitalistische Zerstörung der Stadt hat Mark Purcell im Anschluss an Lefebvre einen Ansatz für eine Neuorientierung der Stadtpolitik hin zu einer radikalen „urban politics of the inhabitant“ (Purcell 2002, 99) entwickelt. Im Zentrum steht dabei das ,Recht auf Stadt‘, das eine Neuordnung der sozialen Beziehungen und des liberal-demokratischen Bürgerschaftsverständnisses begründen soll. Purcell argumentiert, dass Lefebvre in „Das Recht auf Stadt“ auf nicht weniger als die Herauslösung der Stadt aus der überkommenen staatlichen Form politischer Vergemeinschaftung zielt und stattdessen für eine radikaldemokratische Praxis autonomer Selbstorganisation jenseits von Markt und Staat argumentiert, um der neoliberalen Entfremdung entgegenzuwirken. ${ }^{4}$ Die Stadtbewohner (citadins) besitzen demnach ein Recht auf Beteiligung an allen Entscheidungen, die die Gestal-

$4 \quad$ Konkrete Hoffnungen auf die Verwirklichung einer solchen Praxis rebellischer Städte werden von den sogenannten neuen Munizipalismen Süd(ost)europas genährt, die insbesondere in Reaktion auf die Finanzkrise 2008 mit dem Versprechen einer partizipativen und redistributiven Stadtpolitik teils die Rathäuser erobern konnten - so beispielsweise in Barcelona (dazu: Sörensen 2020). 
tung des urbanen Raums betreffen, und ein Recht auf Inbesitznahme dieses Raums (ebd., 102).

Während es dabei um einen radikalen, antistaatlichen Gegenentwurf zur bestehenden Ordnung geht, zielt Kohns Theorie des „urban commonwealth" auf Reformen und Korrekturen im Rahmen der bestehenden liberaldemokratischen Ordnung. Auch sie geht Lefebvre folgend davon aus, dass es sich bei der Stadt und dem städtischen Raum um ein Gemeingut der Stadtbewohnerinnen handelt: „It is the materialization of value that was created collectively by past generations who built the city and diverse contemporaries who repair and animate it“ (Kohn 2016, 3). Das Leitbild der Allmende dient in Kohns Ansatz jedoch nicht dazu, die bestehende Ordnung zu delegitimieren, sondern den Entzug von genuin städtischen Gütern wie Wohnraum, öffentlichem Nahverkehr und öffentlichem Raum überhaupt als Problem politischer Gerechtigkeit zu markieren.

Dem liegt die Annahme zugrunde, dass demokratische Mitgliedschaft stets auch territorial und räumlich vermittelt wird. Die Stadt gewinnt damit politische Bedeutung als Entfaltungsraum individueller und kollektiver Lebensgestaltung sowie politischer Teilhabe. Und zugleich verweist Kohns gerechtigkeitstheoretische Perspektive darauf, dass sich in der Stadt soziale und politische Ungleichheit räumlich materialisiert. Vor diesem Hintergrund betrachtet Kohn ein Recht auf Wohnen und öffentlichen Nahverkehr sowie Mitspracherechte der Bewohnerinnen bei der Umgestaltung der Nachbarschaft als geboten. Dabei überträgt sie einen Gedanken aus der Debatte um territoriale Rechte (Stilz 2019; Moore 2017) auf die Stadt. Demnach haben auch Stadtbewohner neben einem Recht am öffentlichen Raum insgesamt in begrenztem Umfang territoriale Rechte, die ihre jeweiligen, mit dem konkreten Raum verwobenen „located life plans“ (Stilz 2019, 41) schützen. Diese territorialen Rechte nehmen eine Schlüsselrolle im Leitbild der urbanen Allmende ein. Die Begründung dieser Rechte folgt einem Befähigungsansatz: Territoriale Rechte sowie das Recht am öffentlichen Raum schaffen die sozialen Voraussetzungen für die effektive Wahrnehmung von demokratischen Rechten, insbesondere der Kommunikationsrechte wie Meinungs-, Versammlungs- und Demonstrationsfreiheit (Siehr 2019, 670). Sie gewinnen also demokratietheoretische Relevanz, weil sie die Bewohnerinnen überhaupt erst dazu in die Lage versetzen, als Bürger egalitär am politischen Prozess teilzunehmen. 


\section{Zusammenfassung und Ausblick}

Gleichwohl die Stadt als politische Ordnungsform älter ist als der Staat, ist sie im Zuge der modernen Verstaatlichung der Politik weithin aus dem Blickfeld der politischen Philosophie geraten. Unter den Bedingungen der Globalisierung zeichnet sich eine steigende politische Relevanz von Städten ab, die auch die normative Frage nach der demokratischen Legitimität städtischen Handelns neu stellt. Die drei hier vorgestellten Leitbilder der Stadt als Schule der Demokratie, als urbane Kosmopolis und als urbane Allmende präsentieren jeweils politikphilosophische Antworten auf diese Frage, indem sie die Thematisierung der philosophischen und gesellschaftstheoretischen Grundlagen politischer Ordnung aus einer städtischen Perspektive ermöglichen. Allen drei Leitbildern ist ein weiter Demokratiebegriff gemein, der Demokratie als eine erfahrungsbasierte gesellschaftliche Praxis versteht, die sich nicht in institutionell-formalisierten Verfahren wie der Wahl von Bürgermeisterinnen oder Mitgliedern von Stadtparlamenten erschöpft, sondern die individuelle und kollektive Lebensführung als demokratisches Handeln begreift. Im Unterschied zu den vorwiegend repräsentativen Demokratievorstellungen auf staatlicher Ebene setzen alle drei Leitbilder unabhängig von der jeweiligen Theorietradition ein beteiligungsorientiertes Demokratieverständnis voraus. Während das für republikanische, kommunitaristische oder postmarxistische Ansätze zunächst weniger überraschen mag, wird auch in liberalen und kosmopolitischen Ansätze die Interaktion der Individuen im öffentlichen Raum der Stadt als Voraussetzung einer gelingenden Praxis der Differenz benannt.

Die Betonung dieser Agora-Dimension der Demokratie korrespondiert mit einer spezifisch städtischen Vorstellung politischer Mitgliedschaft, die quer zu den gängigen staatszentrierten Mitgliedschaftsmodellen liegt. Während sich dabei die analytische Dreiteilung von liberalen rechtebasierten, kommunitaristischen zugehörigkeitsbasierten und republikanischen partizipationsbasierten Vorstellungen von citizenship durchgesetzt hat (Bellamy, Castiglione und Shaw 2006, 8), verweisen die drei Leitbilder auf die folgenden Kriterien, entlang derer sich eine politische Idee von city-zenship konturiert:

1. Präsenz: Zur politischen Gemeinschaft der Stadt gehört, wer seinen festen Wohnsitz oder dauerhaften Aufenthalt dort hat. Zugehörigkeit in der städtischen politie und darauf aufbauende Ansprüche werden territorial vermittelt. Der Zugang zur Stadt unterliegt keinen Beschränkungen, Städte kennen keine Grenzregime. Damit verbunden ist eine gewisse Dynamik der 
Stadtbürgerschaft, die sich präskriptiver Bedingungen an die Mitgliedschaft somit versperrt. Anders als im Staat, wo Mitgliedschaft vor allem ein rechtlich vermittelter Status ist, soll in der Stadt eine „ethics of presence“ (Bosniak 2019) Teilhabeansprüche rechtfertigen.

2. Öffentlichkeit: Alle drei Leitbilder heben die Bedeutung der Stadt als öffentlicher Raum hervor, in dem sich durch körperliche Präsenz und Begegnung Bürgerinnen als politische Subjekte erfahren und marginalisierte Personen und Gruppen als politische Subjekte konstituieren können. Als Bühne ist der städtische Raum zugleich Austragungsort von politischen und sozialen Konflikten, deren Reichweite über die Stadt hinausgeht.

3. Nähe: Anders als der staatliche ist der städtische politische Raum durch konkrete Erfahrbarkeit gekennzeichnet. Alltägliche Begegnungen heterogener und einander fremder Individuen stellen eine genuin städtische Erfahrung dar. Die Dialektik aus Nähe und sozialer Fremdheit ist eine Voraussetzung dafür, politische Loyalität auf städtischer Ebene anders zu denken als auf staatlicher Ebene. Im Staat als „imagined community“ (Anderson 2017 [1983]) ist Loyalität abhängig von Leitideen, wie sie klassischerweise in der Nation zum Ausdruck kommen, während in der Stadt die physische Nähe auch eine kognitive Nähe und eine gemeinsamen Lebenswelt zu erzeugen in der Lage ist.

Diese drei Kriterien geben Auskunft über die Konstitution eines städtischen demos, von dessen Existenz alle drei Leitbilder ausgehen. Sie eröffnen damit eine Perspektive auf multiple demokratische Mitgliedschaften innerhalb des Staates, die der auf das Volk als einheitliches Legitimationssubjekt fixierte demokratie- und staatstheoretische Fokus verdeckt. Zugleich können darauf aufbauend nun auch die demokratischen Versprechen präzisiert werden, die die drei Leitbilder jeweils mit der Stadt als politischem Handlungsraum verbinden. Im Leitbild der Stadt als Schule der Demokratie steht die Grunderfahrung resonanter Sozialbeziehungen als Voraussetzung der Konstitution demokratischer Subjekte im Vordergrund. Die Erfahrung von Selbstwirksamkeit in sozialen Beziehungen korrespondiert dabei mit der Überzeugung demokratischer Handlungsmacht und der Ausbildung verantwortungsbewusster Bürgerinnen. Das Leitbild der urbanen Kosmopolis reagiert besonders auf die Herausforderung tradierter Demokratievorstellungen durch Migration. Aufgrund des unterstellten produktiven Umgangs mit Differenz erscheint die Stadt als Ermöglichungsraum einer postnationalen Mitgliedschaft sowie der kosmopolitischen Transformation nationaler politischer Identitäten. Das Leitbild der urbanen Allmende schließlich 
begreift Städte als Gegengewichte zu den globalen Kräften des neoliberalen Kapitalismus, die sich vor allem in Form von Immobilieninvestitionen in Städten manifestieren, und als Austragungsorte des Kampfes um soziale Gerechtigkeit. Das Recht auf Stadt lässt sich vor diesem Hintergrund als Reformulierung der Allmende-Idee verstehen und propagiert mit einer neuen Praxis des commoning alternative Eigentumsverständnisse, die sich der Markt- und Investitionslogik widersetzen.

Ob sich vor dem Hintergrund dieser Versprechen eine größere Autonomie von Städten gegenüber dem Staat oder nicht vielmehr eine veränderte politische Praxis in der Stadt rechtfertigen lässt, muss differenziert beurteilt werden. So weist das Leitbild der Stadt als Schule der Demokratie Städten eine funktionale Bedeutung für die Stabilisierung der (staatlichen) Demokratie zu. Der Fokus auf Beteiligung und Selbstwirksamkeit verlangt nach demokratischen Innovationen wie Nachbarschaftsversammlungen, Bürgerräten oder -haushalten, die das Zusammenwirken der Bürgerinnen im politischen Raum der Stadt aktivieren. Mehr Mitsprache an städtischen Angelegenheiten ist nicht gleichbedeutend mit mehr städtischer Autonomie, sodass sich die Vorstellung der Stadt als Schule der Demokratie wohl auch im Rahmen der gegebenen Stadt-Staat-Beziehungen durch institutionelle Innovationen der lokalen Demokratie realisieren ließe.

Im Gegensatz dazu geht das Leitbild der urbanen Kosmopolis von einem zumindest potenziellen Gegensatz zwischen Stadt und Staat aus, insbesondere in den Politikfeldern, in denen erwartet wird, dass Städte eine progressivere, kosmopolitischere Haltung als Nationalstaaten vertreten. Folgt man Bauböck, dann liegt die Zukunft politischer Ordnung in einer „multilevel political architecture“, die auf unterscheidbaren, aber komplementären Mitgliedschaften aufbaut und innerhalb derer die einzelnen Ebenen einander ergänzende Rollen erfüllen (Bauböck 2020). Im Zusammenspiel dieser Ebenen könnten dann Städte, so der Vorschlag Bauböcks, mehr politische Autonomierechte in Bereichen wie Migration, Klima-, Handels- oder sogar Außenpolitik erhalten, die einen Bezug zum transnationalen Charakter des urban citizenship aufweisen (Bauböck 2003, 148f.).

Während also dabei die Aussicht auf die lokale Realisierung kosmopolitischer Werte eine größere Autonomie von Städten gegenüber dem Staat rechtfertigen soll, bleibt das Leitbild der urbanen Allmende ambivalent gegenüber der Frage nach städtischer Autonomie. Aus der gerechtigkeitstheoretisch argumentierenden Perspektive, für die Kohns Ansatz steht, korrespondiert das Recht auf Stadt in erster Linie mit sozialen Teilhaberechten und 
ermöglicht es, Verteilungsgerechtigkeit lokal zu problematisieren. Dabei ist die Stadt Problem und Lösung zugleich: Aufgrund der sozialräumlichen Verdichtung von Ungerechtigkeitserfahrungen erscheint der städtische Raum lebensweltlich besonders geeignet, um Ansprüche auf egalitäre Teilhabe etwa mit Blick auf Mietpreisregulierung, sozialen Wohnungsbau, Spekulationssteuern oder öffentlichen Nahverkehr geltend zu machen. Um ein in diesem Sinne verstandenes Recht auf Stadt durchzusetzen, sind sowohl staatliche als auch städtische Institutionen als Partner gefragt. Der Zentralstaat müsste die rechtlichen und finanziellen Rahmenbedingungen eines Rechts auf Stadt schaffen, die dann von städtischen Institutionen in Kollaboration mit den Bewohnern ausgestaltet würden, wie sie einige Städte etwa bereits erfolgreich in Collaborative-Governance-Experimenten erprobt haben (Foster und Iaione 2020). Demnach steht auch hier weniger die Forderung nach mehr städtischer Autonomie als nach einer veränderten politischen Praxis in der Stadt im Mittelpunkt.

Anders verhält es sich dagegen mit Blick auf die radikaldemokratischen Ansätze der „politics of the inhabitant“, die ebenfalls unter dem Leitbild der urbanen Allmende firmieren. Hier wird die Stadt zum Zentrum demokratischen Handelns und zum primären Raum kollektiver Selbstbestimmung, der die staatliche Ebene gleichsam absorbiert. Außerhalb der Stadt existiert dann nur noch die Weltgesellschaft. Das dahinterstehende politische Ordnungsmodell ist das einer Vielfalt des Lokalen (varieties of localism), deren Gestalt der radikalen Kontingenz ihrer citadins überlassen bleibt: „The right to the city would make these politics possible, but it is the undetermined outcome of these politics that will result in either greater urban democracy or new forms of political domination" (Purcell 2002, 106).

Zusammenfassend kann konstatiert werden, dass eine politische Philosophie der Stadt, die um die städtische Dimension der Demokratie und die Anerkennung einer neuen Rolle von Städten kreist, nicht zwangsläufig mehr städtische Autonomie rechtfertigt - anders als dies in der öffentlichen Stadtdiskussion oftmals insinuiert wird. Bei aller Unterschiedlichkeit liefern die drei Leitbilder indes dringend benötigte Einsichten in einen weithin vergessenen Ort der Demokratie und verweisen auf Lücken staatszentrierter Demokratievorstellungen. Sie markieren Ausgangspunkte, um die Stadt und das Städtische aus einer politikphilosophischen Perspektive neu zu denken und Reformoptionen vor dem Hintergrund bestimmter demokratietheoretischer Vorannahmen zu formulieren. Zugleich offenbaren die drei Leitbilder bestehende Desiderata einer politischen Philosophie 
der Stadt (Frick 2020, 71), wobei ich hier abschließend besonders zwei hervorheben möchte:

Eine auffällige Diskrepanz besteht erstens zwischen dem weithin beobachteten transnationalen Engagement der Städte in den Bereichen Klimaschutz und Ökologie und dem damit korrespondierenden großen Handlungsdruck einerseits und der weitgehenden Nichtbeachtung des Verhältnisses von Stadt und Ökologie in der politischen Philosophie andererseits. In der Stadtforschung besetzen aktuell eher empirisch-technische Untersuchungen von Policy-Optionen und Best-Practice-Beispielen städtischer Resilienz gegenüber den Auswirkungen des Klimawandels das Feld (Keil und Whitehead 2012). Dabei wäre die politische Philosophie besonders geeignet, das spezifische Weltverhältnis von Stadt, städtischer Lebenspraxis und Natur bzw. Ökologie zu durchdringen.

Zweitens verweist gerade die Frage nach mehr städtischer Autonomie, wie sie hier entlang der drei Leitbilder diskutiert wurde, auf die Komplexität der Stadt-Staat-Beziehungen und die Notwendigkeit, diese intensiver politikphilosophisch zu erfassen. Zwar kann die politische Philosophie die Stadt als eigenständigen Raum des Politischen konzeptualisieren, als reale politische Einheiten sind Städte gleichwohl nur in Beziehungen denkbar. Um das transformative Potential von Städten auszuloten, muss die politische Philosophie bei den staatsphilosophischen Grundlagen demokratischer Ordnungen selbst ansetzen und die vielbeschworene Rekonfiguration staatlicher Herrschaft und ihre Folgen für das Verhältnis von Stadt und Staat in den Blick nehmen.

Wenn wir davon ausgehen, dass es einen städtischen demos gibt, stellt sich die Frage nach dem Charakter des Verhältnisses zwischen den multiplen städtischen demoi einerseits und zwischen den partikularen demoi der Stadtbürger und dem übergreifenden demos der Staatsbürgerinnen andererseits. In dem Zusammenhang muss sich die Philosophie der Stadt auch selbstkritisch fragen, ob sie mit der Anerkennung der Stadt als politie eine Differenz stillschweigend normativ perpetuiert, die von der Stadtforschung in den letzten Jahren zunehmend relativiert wurde. Gemeint ist der Stadt-Land-Gegensatz, der sich angesichts der Ubiquität einer städtischen Lebensweise inzwischen eher als Kontinuum darstellt.

All diese Fragen verdeutlichen ein normatives Kernproblem der politischen Philosophie der Stadt, dem bislang noch zu wenig Beachtung geschenkt wird, nämlich das Verhältnis von Partikularität und Egalität innerhalb und zwischen den verschiedenen Einheiten einer politischen Gemeinschaft. Wie 
sind die Ansätze einer politischen Philosophie der Stadt, wie sie hier mit den drei Leitbildern vorgestellt wurden, die zwangsläufig eine gewisse Heterogenität der lokalen Ebene befördern, mit dem zentralen demokratischen Wert staatlich vermittelter politischer Gleichheit vereinbar? Sind innerhalb der politischen Gemeinschaft des Staates Unterschiede etwa hinsichtlich politischer Beteiligungsmöglichkeiten, wohlfahrtsstaatlicher Leistungsniveaus oder Klimaschutzanstrengungen hinnehmbar und in welchem Ausmaß sind sie das? Oder sollten wir womöglich sogar mehr Ungleichheit wagen, um auf diese Weise Chancen für eine Revitalisierung der Demokratie von unten zu eröffnen? Diesen normativen und begrifflichen Fragen muss sich die politische Philosophie der Stadt stellen. Auf diese Weise kann sie einen zentralen Beitrag dazu leisten, herauszufinden, worin das Versprechen der Demokratie heute besteht, welche Wege zu seiner Verwirklichung offenstehen oder erst geschaffen werden müssen und an welchem Ort es am besten einzulösen ist.

\section{Literatur}

Acuto, Michele. 2013. „City Leadership in Global Governance“. Global Governance 19, 481-498.

Amin, Ash. 2002. „Ethnicity and the multicultural city: living with diversity“. Environment and Planning A 34, 959-980.

Anderson, Benedict. 2017 [1983]. Imagined Communities. Reflections on the Origins and Spread of Nationalism. London: Verso.

Aust, Helmut Philipp. 2017. Das Recht der globalen Stadt. Tübingen: Mohr Siebeck.

Barber, Benjamin R. 2013. If mayors rule the world. Dysfunctional nations, rising cities. New Haven: Yale University Press.

Barber, Benjamin R. 2004 [1984]. Strong democracy. Participatory politics for a new age. Berkeley: University of California Press.

Barnett, Clive. 2014. „What do cities have to do with democracy?“. International Journal of Urban and Regional Research 38:5, 1625-1643.

Bauböck, Rainer. 2020. „Cities vs. states: Should urban citizenship be emancipated from nationality?“. Verfassungsblog 2020/1/20, https://verfassungsblog.de/cities-vs-states-should-urban-citizenship-be-emancipated-from-nationality/.

Bauböck, Rainer. 2003. „Reinventing urban citizenship“. Citizenship Studies 7:2, 139-160.

Baumann, Zygmunt. 2013. "Glocalization and Hybridity“. Glocalism: Journal of culture, politics and innovation 1, 1-5. 
Bellamy, Richard, Dario Castiglione und Jo Shaw. 2006. „From national to transnational citizenship“. In Making European Citizens. Civic inclusion in a transnational context, herausgegeben von dies., 1-28. Basingstoke: Palgrave Macmillan.

Blank, Yishai. 2006. „The city and the world“. Columbia Journal of Transnational Law 44, 875-939.

Bickford, Susan. 2000. „Constructing inequality: City spaces and the architecture of citizenship“. Political Theory 28:3, 355-376.

Böckenförde, Ernst-Wolfgang. 1991. „Demokratie als Verfassungsprinzip“. In Staat, Verfassung, Demokratie. Studien zur Verfassungstheorie und zum Verfassungsrecht, 289-378. Frankfurt: Suhrkamp.

Bosniak, Linda. 2019. „Sanctuary and The Contested Ethics of Presence“. https:// ssrn.com/abstract=3238770 or http://dx.doi.org/10.2139/ssrn.3238770 (Zugriff: 26.3.2021)

Boudou, Benjamin. 2020. „Hospitality in Sanctuary Cities“. In The Routledge Handbook of Philosophy of the City, herausgegeben von Sharon M. Meagher, Samantha Noll und Joseph S. Biehl, 279-290. New York: Routledge.

Brenner, Neil. 2019. New Urban Spaces. Urban Theory and the Scale Question. Oxford: Oxford University Press.

Brown, Wendy. 2010. Walled States: Waning Sovereignty. Cambridge, MA: MIT Press.

Castells, Manuel. 2017. Der Aufstieg der Netzwerkgesellschaft. Das Informationszeitalter Wirtschaft - Gesellschaft - Kultur Bd. 1. 2. Aufl. Wiesbaden: Springer VS.

Clüver Ashbrook, Cathryn, und Daniela Haarhuis. 2019. „Retten Städte die UN-Ideale?“. Frankfurter Allgemeine Zeitung, https://www.faz.net/aktuell/politik/ausland/werden-staedte-in-zukunft-staaten-ersetzen-16457571.html?printPagedArticle=true\#pageIndex 2 (Zugriff: 26.3.2021).

Cohen, Jean L. 2014. Globalization and Sovereignty: Rethinking Legality, Legitimacy, and Constitutionalism. Cambrigde: Cambridge University Press.

Davies, Jonathan S., und David L. Imbroscio, Hg. 2010. Critical urban studies. New directions. Albany: State University of New York Press.

Dettling, Daniel. 2019. „Die Probleme der Welt werden in den Metropolen gelöst“. Tagesspiegel, https://www.tagesspiegel.de/politik/intelligenz-der-stadtdie-probleme-der-welt-werden-in-den-metropolen-geloest/25359238.html (Zugriff: 26.3.2021).

Emanuel, Rahm. 2020. The nation city. Why mayors are now running the world. New York: Knopf.

Foster, Sheila R., und Christian Iaione. 2020. „Ostrom in the City: Design Principles and Practices for the Urban Commons“. In Routledge Handbook of the Study of the Commons, herausgegeben von Blake Hudson, Jonathan Rosenbloom, Dan Cole, 235-255. London, New York: Routledge. 
Fraser, Nancy. 2010. Scales of justice. Reimagining political space in a globalized world. New York: Columbia University Press.

Frick, Verena. 2020. „Politische Theorie für das urbane Zeitalter. Ein Plädoyer für die Wiederentdeckung der Städte als demokratische Handlungsräume“. Zeitschrift für Politische Theorie 11:1, 65-74.

Giddens, Anthony. 1990. The Consequences of Modernity. Stanford: Stanford University Press.

Grimm, Dieter. 2009. Souveränität. Herkunft und Zukunft eines Schlüsselbegriffs. Berlin: Berlin University Press.

Habermas, Jürgen. 2011. Zur Verfassung Europas. Ein Essay. Berlin: Suhrkamp.

Hardin, Garrett. 1968. „The tragedy of the commons“. Science 162, 1243-1248.

Haus, Michael. 2002. „Theoretische Überlegungen zum Verhältnis von Bürgergesellschaft und lokaler Politik - zwischen deliberativer Demokratie und kommunitärer Solidarität“. In Bürgergesellschaft, soziales Kapital und lokale Politik herausgegeben von Michael Haus, 76-101. Opladen: Leske und Budrich.

Harvey, David. 2016. Rebellische Städte. 4. Aufl. Berlin: Suhrkamp.

Häußermann, Hartmut, Dieter Läpple und Walter Siebel. 2018. Stadtpolitik. 4. Aufl. Berlin: Suhrkamp.

Held, David. 1997. „Globalization and Democracy“. Global Governance 3, 251-267.

Hidalgo, Anne. 2019. „Paris, Ort des Möglichen“. lettre international N. 127, Winter 2019, 37-52.

Hofmann, Benjamin. 2020. „Whose right to the city? Lessons from the territorial rights debate". In Political Theory and Architecture, herausgegeben von Bernardo Zacka, 197-216. London: Bloomsbury.

Holm, Andrej. 2011. „Das Recht auf die Stadt“. Blätter für deutsche und internationale Politik 8, 89-97.

Huber, Jakob, und Fabio Wolkenstein. 2018. „Gentrification and occupancy rights“. Politics, Philosophy and Economics 17:4, 378-397.

Jörke, Dirk. 2019. Die Größe der Demokratie. Über die räumliche Dimension von Herrschaft und Partizipation. Berlin: Suhrkamp.

Keil, Roger, und Mark Whitehead. 2012. „Cities and the Politics of Sustainability“. In The Oxford Handbook of Urban Politics, herausgegeben von Peter John, Karen Mossberger, und Susan E. Clarke, 520-542. Oxford: Oxford University Press.

Kohn, Margaret. 2016. The death and life of the urban commonwealth. Oxford: Oxford University Press.

Koskenniemi, Martti. 2007. „The fate of public international law: Between technique and politics“. The modern law review 70:1, 1-30.

Krisch, Nico. 2012. Beyond constitutionalism. The pluralist structure of postnational law. Oxford: Oxford University Press. 
Lefebvre, Henri. 2019 [1968]. Das Recht auf Stadt. 3. Aufl. Hamburg: Edition Nautilus.

Middell, Matthias. 2009. „Der Spatial Turn und das Interesse an der Globalisierung in der Geschichtswissenschaft“. In Spatial Turn. Das Raumparadigma in den Kultur- und Sozialwissenschaften, herausgegeben von Jörg Döring und Tristan Thielmann, 103-123. 2. Aufl. Bielefeld: Transcript.

Moore, Margaret. 2017. A political theory of territory. Oxford: Oxford University Press.

Nijman, Janne E. 2016. „Renaissance of the city as a global actor“. In The transformation of foreign policy: drawing and managing boundaries from antiquity to the present, herausgegeben von Gunther Hellmann, Andreas Fahrmeier und Miloš Vec, 209-239. Oxford: Oxford University Press.

Nullmeier, Frank, und Martin Nonhoff. 2010. „Der Wandel des Legitimitätsdenkens“. In Prekäre Legitimitäten. Rechtfertigung von Herrschaft in der postnationalen Konstellation, herausgegeben von Frank Nullmeier et al., 16-44. Frankfurt, New York: Campus.

Offe, Claus. 1975. „Zur Frage der ,Identität der kommunalen Ebene““. In Lokale Politikforschung 2, herausgegeben von Rolf-Richard Grauhan, 303-309. Frankfurt, New York: Campus.

Oomen, Barbara, Martha F. Davis und Michele Grigolo, Hg. 2016. Global Urban Justice. The Rise of Human Rights Cities. Cambridge: Cambridge University Press.

Ostrom, Elinor. 1990. Governing the commons. The evolution of institutions for collective action. Cambridge: Cambridge University Press.

Patberg, Markus. 2018. Usurpation und Autorisierung. Konstituierende Gewalt im globalen Zeitalter. Frankfurt: Campus.

Purcell, Mark. 2002. „Excavating Lefebvre: The right to the city and its urban politics of the inhabitant". Geo Journal 58, 99-108.

Rosa, Hartmut. 2020. Resonanz: Eine Soziologie der Weltbeziehung. 3. Aufl. Berlin: Suhrkamp.

Rosa, Hartmut. 2019. „Demokratie und Gemeinwohl. Versuch einer resonanztheoretischen Neubestimmung“. In Was stimmt nicht mit der Demokratie?, herausgegeben von Hanna Ketterer und Karina Becker, 160-188. Berlin: Suhrkamp.

Rosanvallon, Pierre. 2010. Demokratische Legitimität. Unparteilichkeit - Reflexivität - Nähe. Hamburg: HIS Verlagsgesellschaft.

Sassen, Saskia, Hg. 2007. Deciphering the global. Its scales, spaces and subjects. New York: Routledge.

Sassen, Saskia. 1991. The global cities: New York, London, Tokyo. Princeton: Princeton University Press.

Siebel, Walter. 2018. Die Kultur der Stadt. 3. Aufl. Berlin: Suhrkamp.

Siehr, Angelika. 2016. Das Recht am öffentlichen Raum. Tübingen: Mohr Siebeck. 
Simmel, Georg. 1995 [1903]. „Die Großstädte und das Geistesleben“. In Georg Simmel. Aufsätze und Abhandlungen 1901-19o8, herausgegeben von Rüdiger Kramme, Angela Rammstedt und Otthein Rammstedt, 116-131. Frankfurt: Suhrkamp.

Slaughter, Anne-Marie. 2004. „Disaggregated sovereignty: Towards the public accountability of global government networks“. Government and Opposition 39:2, 159-190.

Smith, Heide Jane M. 2019. „City networks and paradiplomacy as global public policy“. In The Oxford Handbook of Global Policy and Transnational Administration, herausgegeben von Diane Stone und Kim Moloney, 129-147. Oxford: Oxford University Press.

Sörensen, Paul. 2020. „Widerstand findet Stadt. Präfigurative Praxis als transnationale Politik ,rebellischer" Städte“. Zeitschrift für Politische Theorie 10:1, 29-48.

Stilz, Anna. 2019. Territorial sovereignty. A philosophical exploration. Oxford: Oxford University Press.

Sundstrom, Ronald P. 2020. „Residential segregation and rethinking the imperative of integration“. In The Routledge Handbook of Philosophy of the City, herausgegeben von Sharon M. Meagher, Samantha Noll und Joseph S. Biehl, 216-228. New York: Routledge.

Volk, Christian. 2019. „The problem of sovereignty in globalized times“. Law, Culture and the Humanities, online first, https://doi.org/10.1177/1743872119828010.

Von Borries, Friedrich. 2019. „Die gute Stadtluft“. Die Zeit vom 27.12.2019, 41-42.

Wirth, Louis. 1938. „Urbanism as a way of life“. The American Journal of Sociology $44: 1,1-24$.

Wissen, Markus, Bernd Röttger und Susanne Heeg, Hg. 2008. Politics of scale. Räume der Globalisierung und Perspektiven emanzipatorischer Politik. Münster: Westfälisches Dampfboot.

Wollmann, Hellmut. 2002. „Die traditionelle deutsche kommunale Selbstverwaltung - ein ,Auslaufmodell?““. Deutsche Zeitschrift für Kommunalwissenschaften 41:1, 24-51.

Young, Iris Marion. 2000. Inclusion and democracy. Oxford: Oxford University Press.

Young, Iris Marion. 1990. Justice and the politics of difference. Princeton: Princeton University Press.

Young, Iris Marion. 1986. „The ideal of community and the politics of difference“. Social Theory and Practice 12:1, 1-26.

Zürn, Michael. 2011. „Die Rückkehr der Demokratiefrage. Perspektiven demokratischen Regierens und die Rolle der Politikwissenschaft“. In Blätter für deutsche und international Politik 6, 63-74. 